\section{SPINAL CORD}

\section{Ephrins take control}

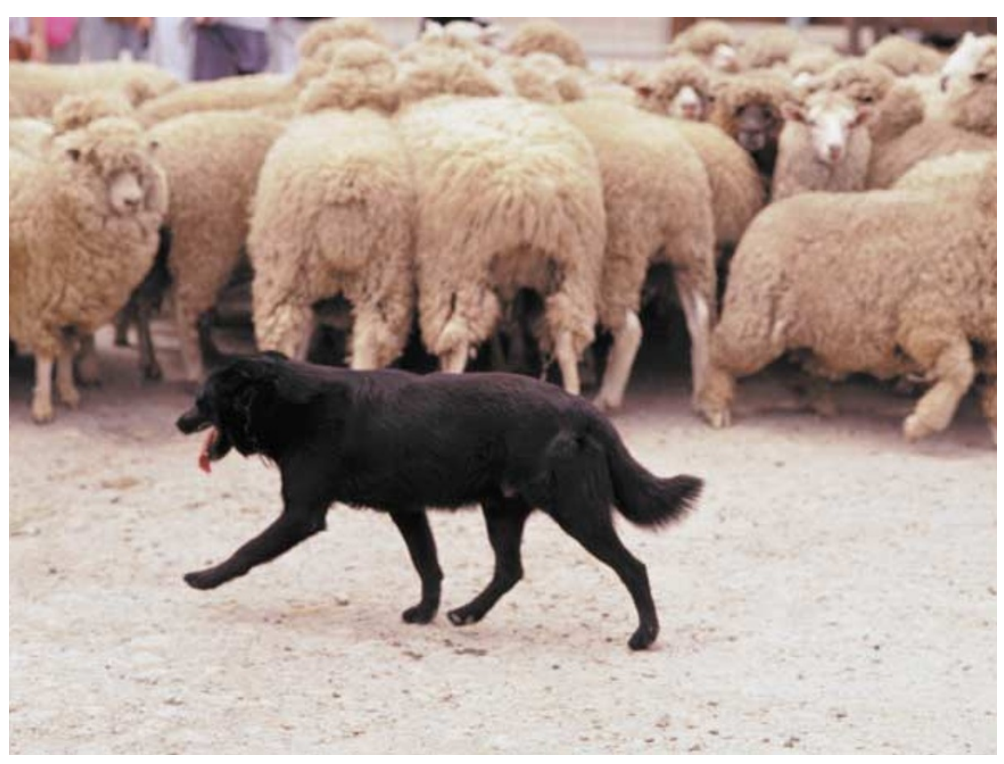

Two new studies shed light on the functions of the Eph receptor tyrosine kinases and their ephrin ligands in the spinal cord. The data highlight roles in pain processing and the control of locomotion, an understanding of which might contribute to the development of therapies for chronic pain and spinal cord injury.

Recently, interactions between EphB and NMDA ( $N$-methylD-aspartate) receptors were shown to regulate synaptic plasticity at glutamatergic synapses in the hippocampus. As NMDA receptors are also key mediators of plasticity in pain-processing regions of the spinal cord, Battaglia and colleagues wondered whether EphB receptors might have a regulatory role in nociceptive pathways.

Activation of postsynaptic EphB receptors on dorsal horn neurons by intrathecal injection of ephrinB2 coupled to an $\mathrm{Fc}$ antibody fragment induced thermal hyperalgesia in adult wild-type rats. Pretreatment with an NMDA receptor antagonist prevented the development of hyperalgesia in ephrinB2-Fc-injected rats, indicating that EphB receptors modulate synaptic transmission through NMDA receptors. Coprecipitation of increased amounts of phosphorylated Src with EphB receptors in ephrinB2-Fc-treated rats implied a role for the Src kinase family in the signalling pathway between EphB and NMDA receptors.

Interactions between NMDA and Eph receptors might also contribute to the control of locomotion, according to a report by Kullander et al. in Science. Addition of serotonin and NMDA to isolated spinal cords of newborn mice produced rhythmic, left-to-right alternating activity in the lumbar segments that control limb movement. By contrast, outputs from the cords of mutant mice lacking either

\title{
Gambling on dopamine
}

Dopamine neurons in the midbrain are thought to produce an error signal that could be important for learning to predict a reward. Now, it seems that the same neurons also signal the level of uncertainty in an experimental trial — which might even give us insight into why gambling is such a popular way to get rid of excess wealth.

Fiorillo and colleagues, writing in Science, describe experiments in which monkeys were conditioned with different visual stimuli, each of which had a different probability of being followed by a reward (a few drops of tasty fruit juice). So, for example, one of the stimuli was always followed by juice, whereas another was followed by juice on only a quarter of the trials. The monkeys learned these relationships - they would lick vigorously at the juice spout when they saw the 'always rewarded' stimulus, and the amount of licking decreased with the probability of reward.

Consistent with earlier work by the same group, dopamine neurons in the monkeys' midbrains produced a stimulus-related signal that was stronger for stimuli that predicted reward more reliably, and a reward-related signal that was stronger when reward had not been reliably predicted. But the authors also saw a new signal - a more gradual increase in firing during the two-second interval between the onset of the stimulus and the potential reward that was greatest when there was the most uncertainty about whether a reward would be forthcoming.

When a stimulus is either always or never associated with reward, there is no uncertainty. By contrast, uncertainty is greatest when the probability of reward is 0.5 , and this was when the sustained response of the dopamine neurons was the greatest. So it seems that, over the course of a trial, the same population of neurons codes two different aspects of the likelihood of reward: one that corresponds to the reward prediction error (shown previously by Waelti et al.), as proposed in formal learning theory by Rescorla and Wagner, and another that measures uncertainty.

This second signal also has correlates in learning theory. According to the Pearce-Hall theory, attention depends on uncertainty about reinforcers, and learning depends on attention. In a real situation, an animal's uncertainty about whether an action or event will be rewarding might just mean that the animal has insufficient information on which to base a prediction - so it will pay off to attend closely to the outcome. The dopamine neurons could be providing a signal that facilitates attention, and therefore learning. Dopamine is closely associated with reward and addiction. The gradual increase in dopaminergic signalling in the presence of uncertainty might not be reinforcing, but if it is it could explain laboratory findings that animals prefer variable reward schedules over fixed ones. It could also, according to the authors, explain why gambling - in which rewards are, by definition, uncertain, and which is hard to explain by other means - is so popular and can even seem to be addictive.

Rachel Jones

(D) References and links ORIGinAL RESEARCH PAPER Fiorillo, C. D. et al. Discrete coding of reward probability and uncertainty by dopamine neurons. Science 299, 1898-1902 (2003)

FURTHER READING Schultz, W. Multiple reward signals in the brain. Nature Rev. Neurosci. 1, 199-207 (2000) | Waelti, P. et al. Dopamine responses comply with basis assumptions of formal learning theory. Nature 412, 43-48 (2001) 